\title{
Determinación de factores de riesgo sanitario ambiental para selección de area del relleno sanitario en la localidad de Palpa
}

\section{Determination of sanitary risk factors for area selection from the sanitary filling in the locality of Palpa}

\author{
Ramiro Zuzunaga Morales \\ razumor161@yahoo.es - Universidad Nacional San Luis Gonzaga \\ https://orcid.org/0000-0003-3527-2404 \\ Adolfo Amadeo Ramírez Zegarra \\ Universidad Nacional San Luis Gonzaga
}

Recibido el 21/12/20 | Aceptado el 15/01/21

DOI: https://doi.org/10.47190/nric.v3i1.140

\section{Resumen}

La gestión de los residuos sólidos sin distinción de su origen o lugar geográfico de generación se convierte en un problema que cada vez nos llama a buscar soluciones de la mano con la tecnología y las buenas prácticas por parte de los generadores, pues a consecuencia de los incrementos de las cantidades de residuos sólidos que se vienen generando, se necesitan infraestructuras de manejo y disposición de residuos sólidos que aseguren tratamientos adecuados con la finalidad de proteger el ambiente.

La actividades del Manejo de residuos sólidos y su disposición final en la provincia de Palpa viene originando problemas de polución y contaminación en el sector de Llipata, este escenario ambiental característico de nuestra región donde cada vez es más importante específicamente por el crecimiento de la actividades económicas y crecimiento poblacional, agudizándose por el arrojo de grandes volúmenes de residuos urbanos, que origina diversos problemas ambientales debido a la inadecuada disposición final de las grandes cantidades de material residual producido que son vertidos directamente a los cuerpos de agua y al suelo sin protección alguna.

El presente trabajo permitirá determinar los factores de riesgo sanitario y ambiental en el espacio geográfico del sector de Pajonal de la Provincia de Palpa, Región Ica.

Palabras claves: Factores de Riesgo, Área, Relleno Sanitario, Botadero, Residuos Sólidos.

\begin{abstract}
The management of solid waste without distinction of its origin or geographic place of generation becomes a problem that increasingly calls us to seek solutions hand in hand with technology and good practices by generators, as a result of the Increases in the amounts of solid waste that are being generated, require solid waste management and disposal infrastructures that ensure adequate treatments in order to protect the environment.
\end{abstract}

The activities of solid waste management and its final disposal in the province of Palpa has been causing pollution and pollution problems in the sector of Llipata, this environmental scenario characteristic of our region where it is increasingly important specifically for the growth of economic activities and population growth, exacerbated by the splash of large volumes of urban waste, which causes various environmental problems due to the inadequate final disposal of large amounts of waste material produced that are discharged directly to bodies of water and soil without any protection.

The present work will allow to determine the sanitary and environmental risk factors in the geographic space of the sector of Pajonal of the Province of Palpa, Ica Region.

Keywords: Risk Factors, Area, Sanitary Landfill, Dump, Solid Waste. 


\section{Introducción}

El presente trabajo de investigación permite determinar los factores de riesgo sanitario y ambientales para la selección de area del relleno sanitario de la localidad de Palpa con el objetivo de optar como probable geografía para la Selección de Área de Relleno Sanitario, se desarrolla según el marco del "Programa de Desarrollo de Sistemas de Gestión de Residuos Sólidos en zonas prioritarias" a fin de continuar con el proceso de adecuamiento a la Ley General de Residuos Sólidos N ${ }^{\circ} 27314$ y la metodología planteada en el presente documento considera los criterios del Reglamento de la Ley de Residuos Sólidos (DS 057-2004/PCM) establecidos en el Art. 67, y del apéndice $N^{\circ} 4$ Selección de Sitio para Rellenos Sanitarios, de la Guía de Identificación, Formulación y Evaluación Social de Proyectos de Residuos Sólidos Municipales a Nivel de Perfil. El desarrollo de cada uno de los criterios establecidos en las Normas mencionadas anteriormente, permitirá conocer si el lugar es técnica, ambiental y socialmente adecuado para la instalación de la infraestructura de manejo de residuos sólidos y generar condiciones de salubridad e higiene que contribuyan a elevar la calidad de vida de la población local.
En el trabajo de investigación se evalúan las características del área del botadero de Llipata y contrastarlo con las condiciones apropiadas para una infraestructura de disposición final y aprovechamiento de residuos sólidos, acorde con las normas técnicas y los documentos legales pertinentes.

\section{Materiales y metodos}

\section{Materiales}

Se utilizaran

- Planos, mapas y cartas geográficas.

- GPS.

- Winchas de Medición

- Cámaras Fotográficas,

- Equipo de Estación Total

\section{Métodos}

Dentro del método de selección del área en estudio, se tomaron los criterios de selección, ponderación y de valoración mostrados en la tablas siguientes.

\section{Criterios de seleccion}

\begin{tabular}{|c|c|c|c|}
\hline \multirow{2}{*}{ ITEM } & \multirow{2}{*}{ CRITERIOS DE SELECCIÓN } & \multirow{2}{*}{$\begin{array}{c}\text { Ley } \\
27314\end{array}$} & Alternativa № 1 \\
\hline & & & LLIPATA \\
\hline 1 & $\begin{array}{l}\text { Uso actual del suelo - compatibilidad con el uso actual y } \\
\text { planes urbanos }\end{array}$ & CE & Terreno eriazo \\
\hline 2 & $\begin{array}{l}\text { Área considerada en el plan Integral de gestión Ambiental } \\
\text { de Residuos Sólidos }\end{array}$ & CE & Sí \\
\hline 3 & $\begin{array}{l}\text { Tamaño del terreno o Superficie disponible para rellenar } \\
\text { (ha) }\end{array}$ & CG & 15.00 has \\
\hline 4 & Vida útil (años) & $\mathrm{CE}$ & $>5$ años \\
\hline 5 & Pasivos ambientales & $\mathrm{CE}$ & No \\
\hline 6 & $\begin{array}{l}\text { Distancia a fuentes de aguas superficiales }(\mathrm{m}) \text { medidas } \\
\text { en línea recta }\end{array}$ & $\mathrm{NC}$ & $10.0 \mathrm{Km}$. \\
\hline 7 & $\begin{array}{l}\text { Distancia a fuentes de abastecimiento de agua sub } \\
\text { superficiales } .(m) \text { bombeo }\end{array}$ & $\mathrm{NC}$ & $>5 \mathrm{~km}$ \\
\hline 8 & Opinión Pública & $\mathrm{CE}$ & Favorable \\
\hline 9 & Barrera Sanitaria & $\mathrm{CE}$ & $\mathrm{SI}$ \\
\hline 10 & Posibilidad del material de cobertura & $\mathrm{CE}$ & BUENA \\
\hline 11 & Profundidad del nivel freático $(\mathrm{m})$ & CG & + de 60m aprox. \\
\hline 12 & $\begin{array}{l}\text { Condiciones meteorológicas del sitio (principalmente pp } \\
\text { anual) }\end{array}$ & CG & $\begin{array}{l}\text { Media anual de } 30 \\
\mathrm{~mm} / \mathrm{año}\end{array}$ \\
\hline 13 & Permeabilidad de suelo (cm/seg.) & & $\begin{array}{c}1 \text { a } 5 \times 10(-4) \mathrm{cm} / \mathrm{seg} \\
\text { aprox. }\end{array}$ \\
\hline 14 & Pendiente \% (Topografía del Terreno) & CG & $1 \%$ \\
\hline 15 & Dirección predominante del viento & CG & W-E \\
\hline 16 & Distancia a la población $(\mathrm{Km})$ centro & $\mathrm{CE}$ & $10 \mathrm{Km}$. \\
\hline 17 & Distancia a granjas crianza de animales $(\mathrm{m})$ & $\mathrm{CE}$ & $10 \mathrm{~km}$ \\
\hline 18 & Área arqueológica & $\mathrm{CE}$ & $10 \mathrm{Km}$. \\
\hline 19 & Área natural protegida por el estado & CE & No \\
\hline 20 & Vulnerabilidad a desastres (inundaciones) & CE & No \\
\hline 21 & Propiedad del terreno & $\mathrm{CE}$ & Bienes Nacionales \\
\hline 22 & Impacto de tránsito vehicular sobre la comunidad & $\mathrm{NC}$ & Mínimo \\
\hline 23 & $\begin{array}{l}\text { Accesibilidad al sitio (distancia desde la vía de acceso } \\
\text { principal } \mathrm{Km} \text {.) }\end{array}$ & $\mathrm{NC}$ & $8.0 \mathrm{Km}$. \\
\hline 24 & Distancia a aeropuertos o pistas aterrizaje & $\mathrm{CE}$ & $40.0 \mathrm{Km}$. \\
\hline
\end{tabular}

CE: considerado de manera específica señalando valores

CG: considerado de manera general

NC: no considerado en forma específica 


\section{Tabla de ponderación}

\begin{tabular}{|c|c|c|c|}
\hline \multirow{2}{*}{ Ítem } & \multirow{2}{*}{ Criterios de selección } & Alternativa A & $\begin{array}{c}\text { Factor de } \\
\text { Ponderación }\end{array}$ \\
\hline & & LLIPATA & \\
\hline 1 & $\begin{array}{l}\text { Uso actual del suelo - compatibilidad con el uso actual y } \\
\text { planes urbanos }\end{array}$ & 5 & 5.0 \\
\hline 2 & Área considerada en el plan de gestión & 3 & 5.0 \\
\hline 3 & $\begin{array}{l}\text { Tamaño del terreno o Superficie disponible para rellenar } \\
\text { (ha) }\end{array}$ & 5 & 3.0 \\
\hline 4 & Vida útil (años) & 4 & 3.0 \\
\hline 5 & Pasivos ambientales & 4 & 3.0 \\
\hline 6 & $\begin{array}{l}\text { Distancia a fuentes de aguas superficiales }(m) \text { medidas } \\
\text { en línea recta }\end{array}$ & 4 & 3.0 \\
\hline 7 & $\begin{array}{l}\text { Distancia a fuentes de abastecimiento de agua } \\
\text { subsuperficiales .(m)bombeo }\end{array}$ & 3 & 3.0 \\
\hline 8 & Opinión Pública & 3 & 7.0 \\
\hline 9 & Barrera Sanitaria & 4 & 5 \\
\hline 10 & Posibilidad del material de cobertura & 3 & 3.0 \\
\hline 11 & Profundidad del nivel freático $(\mathrm{m})$ & 4 & 3.0 \\
\hline 12 & $\begin{array}{l}\text { Condiciones meteorológicas del sitio (principalmente pp. } \\
\text { anual) }\end{array}$ & 4 & 3.0 \\
\hline 13 & Permeabilidad de suelo (cm/seg.) & 3 & 3.0 \\
\hline 14 & Pendiente \% (Topografía del Terreno) & 3 & 2.0 \\
\hline 15 & Dirección predominante del viento & 3 & 3.8 \\
\hline 16 & Distancia a la población (Km) & 3 & 6.0 \\
\hline 17 & Distancia a granjas crianza de animales $(\mathrm{m})$ & 2 & 6.0 \\
\hline 18 & Área arqueológica & 5 & 4.0 \\
\hline 19 & Área natural protegida por el estado & 5 & 4.0 \\
\hline 20 & Vulnerabilidad a desastres naturales & 4 & 5.0 \\
\hline 21 & Propiedad del terreno & 4 & 5.0 \\
\hline 22 & Impacto de tránsito vehicular sobre la comunidad & 4 & 2.0 \\
\hline 23 & $\begin{array}{l}\text { Accesibilidad al sitio (distancia desde la vía de acceso } \\
\text { principal } \mathrm{Km} \text {.) }\end{array}$ & 5 & 3.0 \\
\hline 24 & Distancia a aeropuertos o pistas aterrizaje & 2 & 3.0 \\
\hline
\end{tabular}

Valores para Ponderación

\begin{tabular}{|l|c|}
\hline \multicolumn{1}{|c|}{ Calificación } & Valor \\
\hline Muy Malo & 1 \\
\hline Malo & 2 \\
\hline Regular & 3 \\
\hline Bueno & 4 \\
\hline Excelente & 5 \\
\hline
\end{tabular}


Tabla de valoracion de alternativas

\begin{tabular}{|c|c|c|}
\hline \multirow{2}{*}{ Item } & \multirow{2}{*}{ Criterios de selección } & Alternativa № 1 \\
\hline & & Pampa Satelite" \\
\hline 1 & Uso actual del suelo - compatibilidad con el uso actual y planes urbanos & 25 \\
\hline 2 & Área considerada en el plan de gestión & 15 \\
\hline 3 & Tamaño del terreno o Superficie disponible para rellenar (ha) & 15 \\
\hline 4 & Vida útil (años) & 12 \\
\hline 5 & Pasivos ambientales & 12 \\
\hline 6 & Distancia a fuentes de aguas superficial $(\mathrm{m})$ & 12 \\
\hline 7 & Distancia a fuentes de abastecimiento de agua subsuperficiales.$(\mathrm{m})$ bombeo & 9 \\
\hline 8 & Opinión Pública & 21 \\
\hline 9 & Barrera Sanitaria & 20 \\
\hline 10 & Posibilidad del material de cobertura & 9 \\
\hline 11 & Profundidad del nivel freático $(\mathrm{m})$ & 12 \\
\hline 12 & Condiciones meteorológicas del sitio (principalmente pp anual) & 12 \\
\hline 13 & Permeabilidad de suelo (cm/seg.) & 9 \\
\hline 14 & Pendiente \% (Topografía del Terreno) & 6 \\
\hline 15 & Dirección predominante del viento & 11.4 \\
\hline 16 & Distancia a la población $(\mathrm{Km})$ & 12 \\
\hline 17 & Distancia a granjas crianza de animales $(\mathrm{m})$ & 12 \\
\hline 18 & Área arqueológica & 30 \\
\hline 19 & Área natural protegida por el estado & 20 \\
\hline 20 & Vulnerabilidad a desastres naturales & 20 \\
\hline 21 & Propiedad del terreno & 20 \\
\hline 22 & Impacto de tránsito vehicular sobre la comunidad & 8 \\
\hline 23 & Accesibilidad al sitio (distancia desde la vía de acceso principal Km.) & 15 \\
\hline 24 & Distancia a aeropuertos o pistas aterrizaje & 6 \\
\hline & Total Valoración & 343.40 \\
\hline
\end{tabular}

\section{Resultados}

Los resultados de los factores de riesgo determinan el espacio geográfico de coordenadas geográficas siguientes:

\section{Ubicación geográfica de area}

\begin{tabular}{|l|c|c|c|}
\hline \multirow{2}{*}{ Áreas alternativas } & \multirow{2}{*}{ Vértices. } & \multicolumn{2}{|c|}{ Coordenadas UTM } \\
\cline { 3 - 4 } & & Este & Norte \\
\hline \multirow{3}{*}{ BOTADERO LLIPATA } & $\mathrm{A}$ & 480520.00 & 8385510.00 \\
\cline { 2 - 4 } & $\mathrm{B}$ & 480490.00 & 8385890.00 \\
\cline { 2 - 4 } 15 has & $\mathrm{C}$ & 480990.00 & 8385920.00 \\
\cline { 2 - 4 } & $\mathrm{D}$ & 481050.00 & 8385560.00 \\
\hline
\end{tabular}

\section{Discusion}

Del análisis a los factores de riesgo sanitario ambiental para el diseño del relleno sanitario, en el área del actual botadero de LLIPATA es la de mejor posibilidad, dada su posición geográfica, alejada a una distancia conveniente de la ciudad, facilidad en el acceso (Carretera Panamericana Sur),

El material de cobertura del lugar donde se encuentra el área de Llipata, es material arcilloso arenoso, aspecto que evitará el material de préstamo y gastos por transporte de material de cobertura.

Las áreas aledañas a la zona del botadero de Llipata, presentan una morfología y topografía adecuada y con las características del suelo que tienen alta porosidad.
Es necesario realizar estudios complementarios de línea base de las condiciones ambientales actuales en la zona en estudio.

\section{Conclusiones}

Se concluye que del análisis a los factores de riesgo sanitario ambiental para el diseño del relleno sanitario, en el área del actual botadero de LLIPATA es la de mejor posibilidad, dada su posición geográfica, alejada a una distancia conveniente de la ciudad, facilidad en el acceso (Carretera Panamericana Sur),

El material de cobertura del lugar donde se encuentra el área de Llipata, es material arcilloso arenoso, aspecto que evitara el material de préstamo y gastos por transporte de material de cobertura.

Las áreas aledañas a la zona del botadero de Llipata, presentan una morfología y topografía adecuada y con las características del suelo que tienen alta porosidad.

No existen viviendas a distancias menores a más 8 kilómetros aproximadamente.

No existe evidencia de riesgos y vulnerabilidad ante desastres naturales que pudieran comprometer la estabilidad de la infraestructura sanitaria futura. 


\section{Referencias bibliográficas}

Instituto Nacional de Estadística e Informática. 1996. III Censo Nacional Agropecuario. Resultados definitivos. Perú: compendio estadístico, $\mathrm{n}^{\circ} 26$ Tomo II, Ministerio de Agricultura. Lima. 1567 pp.

EI PNUD apoya enérgicamente la recomendación de la Conferencia Internacional sobre Agua Dulce celebrada en Bonn en diciembre de 2001, de que el objetivo sobre el agua potable se complemente mediante la meta correspondiente de reducir a la mitad la proporción de personas que carece de acceso a mejores servicios de saneamiento para 2015.

Departamento de Saneamiento Ambiental "Mejoramiento de la disposición final de los desechos sólidos en el relleno sanitario del valle - Cuenca". México, 1995.

DIGESA, Marco Institucional de los Residuos Sólidos en el Perú, Perú, 2004. 126 pág. Empresa de Servicio de Limpieza Municipal Pública del Callao, "Proyecto de Recolección y Disposición Final de los Residuos Sólidos en el Callao". Perú ,1993.

JICA, A. d. (30 de mayo de 2016). pwi. Obtenido de https://pwi.com.pe/gestion-de-residuossolidos-2/ Ambiente, M. d. (23 de diciembre de 2016).

Decreto Legislativo № 1278 - DECRETO LEGISLATIVO QUE APRUEBA LA LEY DE GESTION INTEGRAL DE RESIDUOS SOLIDOS. EL PERUANO, pag. 17. Ambiente, M. d. (21 de diciembre de 2017).

Reglamento del Decreto Legislativo № 1278, Decreto Legislativo que aprueba la Ley de Gestión Integral de Residuos

Bacner, B. A. (2016). GESTIÓN DE LOS RESIDUOS SÓLIDOS Y EL IMPACTO AMBIENTAL EN EL PUEBLO JOVEN 9 DE OCTUBRE CHICLAYO, 2016. Chiclayo: 1.

Brandan, I. R. (2012). Guía de Diseño, construcción, operación, mantenimiento y cierre de relleno sanitario. Lima: Ministerio del Ambiente.

Cruz, M. I. (2017). Estrategias aplicadas para el cierre de 30 Botaderos de Residuos Sólidos en Perú. Lima: 1. desarrollo, B. I. (6 de febrero de 2017). BID. Obtenido de https://www.iadb.org/es/project/PE-L1153

Jaime, C. R. (2007). MEJORAMIENTO DE LA GESTIÓN DE LOS RESIDUOS SÓLIDOS EN EL AMBITO. Pasco - Huariaca: 1. Minam. (5 de Mayo de 2018). SINIA. Obtenido de http://sinia.mma.gob.cl/

Recuperación de áreas Degradada por Residuos Sólidos en el Distrito de Coracora, Provincia Parinacochas, Región de Ayacucho. Lima: 1.

Ambiente, D. A. (2009). Ministerio del Ambiente . San Isidro, Lima, Perú : Consorcio Ciudad Saludable - Perú Waste Innovation S.A.C.

Antonio Brack Egg. (2009). Menisterio del ambiente. Lima. Lima: Perú Waste Innovation S.A.C.

Bolaños.g, y. (2007). Diagnóstico de los residuos sólidos y líquidos de la comunidad plan de la laguna, Reserva Natural Laguna de Apoyo. Nicaragua: tesis .

Bustamante alsina jorge. (1995). Derecho ambiental fundamentacion y normativa. Buenos Aires:
Abeledo Perrot. Care, M. D. (2001). "Proyecto agua: acceso, gestión y uso racional del agua".

Carmen, J. P. (2003). Las competencias locales en materia de residuos solidos. Madrid: Civitas. 